\title{
Un changement espéré dans la formation pharmaceutique en France : le point de vue d'internes en pharmacie français
}

\section{The opinion of pharmacy interns in France on pharmacy education expectations}

Aurélie GUÉRIN ${ }^{1}$, Marie Élaine MÉTRAS ${ }^{1}$, Delphine MERGER ${ }^{1}$, Eve COURBON ${ }^{1}$, Jean-François BUSSIÈRES ${ }^{1,2}$

1 Unité de recherche en pratique pharmaceutique, département de pharmacie, CHU Sainte-Justine, Montréal, Québec, Canada

2 Faculté de Pharmacie, Université de Montréal, Montréal, Québec, Canada

Manuscrit soumis à la rédaction le 18 août 2013 ; commentaires éditoriaux formulés aux auteurs le 9 et le 14 mars 2014 ; accepté pour publication le 15 mars 2014

Mots-clés

Pharmacie ;

formation

universitaire :

Canada ; Europe ;

France ; réformes

curriculaires

\section{Messages clés}

- La pratique pharmaceutique a considérablement évolué au cours des deux dernières décennies et une révision des dispositifs curriculaires en formation initiale en pharmacie s'impose pour mieux répondre aux besoins de la population.

- Le projet de loi français pour l'enseignement supérieur et la recherche du 3 juillet 2013 et la directive européenne 2013/55/EU sur le principe commun de formation encouragent la France à une ouverture internationale.

- Une étude comparée des dispositifs de formation pharmaceutique initiale en vigueur au Québec, en Europe et en France, permet d'identifier de nouveaux défis pédagogiques à relever pour la formation initiale en pharmacie. Ils concernent respectivement l'évolution de la formation en fonction des besoins de la population, le renouvellement des méthodes pédagogiques, l'intégration des ressources numériques, la place de l'étudiant au sein de sa formation et l'organisation des stages.

\section{Keywords}

Pharmacy; academic training; Canada;

\section{Key messages}

- Over the last two decades, pharmacy practice has evolved, and changes in pharmacy education are required in order to meet population needs. 
Europe; France; curricular revision
- A French draft legislation on graduate studies and research and the 2013/55/EU European directive on the common training framework will both help bring about international harmonization. Knowledge of other international training frameworks and a review of French education will provide food for thought.

- In this article, we sum up the Quebec, European and French pharmacy undergraduate education programs and discuss teaching challenges in pharmacy. The progress of education in step with population needs, renewed teaching methods, the integration of digital resources, student involvement and internship organization are also examined.

\section{Introduction}

Internes français en pharmacie hospitalière en stage au Québec au Centre hospitalier universitaire SainteJustine et à l'unité de recherche en pratique pharmaceutique, nous avons pris conscience des différences relatives à la formation pharmaceutique entre la France et le Québec. Dans le contexte d'adoption par le parlement français de la Loi pour l'enseignement supérieur et la recherche ${ }^{[1]}$, le 3 juillet 2013 , de l'adoption de la directive européenne 2013/55/EU le 20 novembre 2013, relative au principe commun de formation $^{[2]}$, nous saisissons l'opportunité de notre exposition à l'enseignement et à la pratique pharmaceutique québécois pour faire écho à ces nouvelles dispositions réglementaires et discuter quelques différences pédagogiques relatives à la formation initiale en pharmacie, respectivement en France et au Québec.

Le projet de loi français et la directive européenne encouragent en effet la France à intégrer une perspective d'ouverture internationale. Dans cette perspective, une analyse comparée et critique de programmes de formation implantés ailleurs dans le monde peut apporter une contribution utile à cette réflexion et à la révision curriculaire à mettre en œuvre pour répondre davantage aux besoins de la population et du système de santé [3-8] $^{[3}$.

\section{Évolution de la profession de pharmacien : I'essor de la pharmacie clinique}

La pratique pharmaceutique a considérablement évolué au cours des deux dernières décennies. Les dispositifs de formation initiale des pharmaciens devraient dès lors évoluer pour prendre en compte la redéfinition de ces pratiques professionnelles en pharmacie, afin de mieux répondre aux besoins de la population. La Fédération internationale de pharmacie et de nombreux organismes du domaine de la santé, à l'échelle mondiale, reconnaissent le rôle clinique prédominant du pharmacien et la nécessité de sa contribution pour favoriser l'atteinte de meilleurs résultats de santé, compte tenu de l'importance croissante de la pharmacothérapie, de ses risques et de ses coûts. Ce champ d'intervention professionnelle est désigné par le terme de pharmacie clinique ; il s'adosse à une philosophie de l'approche des soins pharmaceutiques, au regarde de laquelle le pharmacien doit établir une relation de confiance avec le patient, identifier, prévenir et résoudre les problèmes liés à la pharmacothérapie, tout en assurant une surveillance proactive de cette thérapie médicamenteuse afin d'atteindre les objectifs pharmaco thérapeutiques fixés de façon interdisciplinaire ${ }^{[9]}$.

\section{État des lieux synthétique de la formation pharmaceutique initiale au Québec, en Europe et en France}

\section{Au Québec, en contexte nord-américain}

Le programme de baccalauréat en pharmacie de l'Université de Montréal a fait l'objet d'une refonte entière, qui a aboutit à la mise en place, en 2007, du nouveau programme de doctorat professionnel en pharmacie (Pharm. D), dans l'esprit des révisions de 
programme nord-américaines entreprises au cours des années quatre-vingt-dix ${ }^{[10,11]}$. La Faculté de pharmacie de l'Université de Montréal est la première au Canada à offrir ce nouveau programme de doctorat professionnel de premier cycle ${ }^{[12-14]}$.

$\mathrm{Au}$ Québec, dans le cadre de la mise en place du programme de Doctorat professionnel en pharmacie, des principes ont été énoncés, notamment en regard de l'architecture générale du programme : 1) chaque année d'études du programme doit être guidée par une thématique signifiante pour l'étudiant, le faisant évoluer d'un contexte relativement restreint (soins aux patients) à une perspective plus large (interdisciplinarité, continuité des soins, activités de promotion et de prévention de la santé) ; 2) la majorité des cours se rapportant aux compétences disciplinaires doit être structurée en fonction des systèmes physiologiques. Les connaissances ainsi organisées seront d'accès plus faciles lors de l'exercice de la profession ; 3) la structure doit favoriser l'interdisciplinarité afin de permettre à l'étudiant de se préparer à exercer son rôle au sein d'une équipe multidisciplinaire et à assurer la continuité des soins ; 4) des cours doivent être développés spécifiquement dans le but de faciliter l'intégration des compétences tant spécifiques que transversales ${ }^{[12]}$.

Quatre thématiques annuelles ont été adoptées : pour la première année, s'intégrer dans un milieu de pratique ; pour la deuxième année, prodiguer des soins pharmaceutiques dans un contexte multidisciplinaire ; pour la troisième année, prodiguer des soins pharmaceutiques et offrir des services dans son milieu et dans sa communauté et, pour la quatrième année, intégrer les compétences dans une pratique professionnelle. Chaque année comporte au moins quatre des six modules suivants : 1) le médicament et l'homme (acquisition de connaissances en sciences biologiques, pharmaceutiques et cliniques, fondée sur l'étude des systèmes physiologiques) ; 2) le médicament et la société, (application à la pharmacie des sciences de la communication, des sciences des populations et de la gestion) ; 3) les laboratoires (mise en pratique des habiletés développées dans le cadre des modules précédents) ; 4) les activités d'intégration (conception, réalisation, implantation et évaluation d'un projet d'envergure autour d'une problématique sociale complexe, afin de rendre un service à la communauté, et formation à la collaboration en sciences de la santé) ; 5) les stages ; 6) les cours à option.

Ce canevas traduit le projet, au premier trimestre, d'une approche intégrée par système physiologique, réunissant les cours d'anatomie, de physiologie, de biochimie, de biologie cellulaire et moléculaire et de nutrition. Au deuxième trimestre, trois cours intègrent la chimie médicinale, la physicochimie, la galénique, la pharmacogénétique, la pharmacocinétique et la pharmacodynamie. Quatorze cours de soins pharmaceutiques intègrent les notions de physiopathologie, d'évaluation de l'état physique, de biologie clinique, de galénique appliquée, de pharmacologie, de cinétique clinique, de toxicologie et de thérapeutique appliquée. Cette refonte met en évidence la dominance des cours à portée clinique sur l'ensemble des quatre années de la formation. Dès les deux premières années de sa formation, l'étudiant québécois est exposé à des cours de soins pharmaceutiques sur des thématiques telles que l'immunologie, l'hématologie, la néphrologie, l'infectiologie, la gastro-entérologie, la cardiologie.

\section{En Europe}

De nombreuses différences existent en Europe quant à la formation initiale des pharmaciens. Les résultats du projet PHARMacy education IN Europe (PHARMINE), fondé par la commission européenne, font état de ces nombreuses différences pédagogiques $^{[15]}$. Ces différences sont actuellement discutées en Europe dans le cadre de l'adoption de la directive européenne 2013/55/EU, sur le principe commun de formation, afin de faciliter la mobilité professionnelle en Europe ${ }^{[2]}$. En tout état de cause, la formation européenne demeure cependant traditionnelle, axée davantage sur les sciences fondamentales.

Le cas plus spécifique de la France

En France, le Ministère de l'enseignement supérieur et de la recherche définit onze champs 
d'enseignements en pharmacie à savoir : 1) la biodiversité et la bio-évolution des règnes animal, végétal et fongique ; 2) les voies d'accès aux substances actives médicamenteuses ; 3) les sciences biologiques (biologie cellulaire et moléculaire, génétique, biochimie, microbiologie, parasitologie, physiologie, hématologie, immunologie) ; 4) les sciences analytiques (techniques d'analyses physiques, chimiques et biologiques) ; 5) le cycle de vie du médicament et le circuit pharmaceutique ; 6) les sciences pharmacologiques (pharmacologie moléculaire et générale, pharmacocinétique) ; 7) la formulation, la fabrication et les aspects biopharmaceutiques des médicaments ; 8) la qualité des produits de santé ; 9) les pathologies, les sciences biologiques et thérapeutiques (sémiologie clinique et biologique, chimie thérapeutique, pharmacognosie, biologie clinique, pharmacologie des substances actives, stratégies thérapeutiques, pharmacie clinique, iatrogénèse et toxicité) ; 10) la santé publique et 11) les statistiques.

Il existe des différences importantes en termes de contenus et d'importance relative des différents thèmes entre le programme québécois et le programme français, même si les patients de ces deux environnements géopolitiques sont traités avec le même arsenal thérapeutique et ont des besoins similaires en pharmacothérapie. La prédominance donnée aux sciences cliniques et à la formation d'un pharmacien clinicien capable de prodiguer des soins pharmaceutiques directs à tous ses patients n'est pas étrangère à la présence de professeurs de clinique, c'est à dire exerçant une pratique clinique et dont le parcours académique est centré sur l'exercice de la pharmacie, l'enseignement et la réalisation d'activités créatrices et non sur la recherche financée. Au 31 mars 2013, la Faculté de pharmacie de l'Université de Montréal compte 33 professeurs de carrière (parcours $\mathrm{Ph} \mathrm{D}+$ formation post-doctorale) et 16 professeurs de clinique (parcours Pharm. D. + Maitrise en sciences -M. Sc.- et formation clinique complémentaire). La formation française demeure axée davantage sur les sciences fondamentales et la capacité de former des pharmaciens désirant poursuivre un cursus de recherche. Si l'enseignement des sciences fondamentales demeure nécessaire à la formation des cliniciens en santé, sa juste part doit être évaluée pour assurer une maîtrise des connaissances et compétences menant à la formation d'un clinicien.

\section{Les nouveaux défis pédagogiques à relever pour la formation initiale en pharmacie}

Le 3 juillet 2013, le parlement français a adopté la Loi pour l'enseignement supérieur et la recherche ${ }^{[1]}$. Cette loi découle d'une vaste consultation lancée en juillet 2012 auprès de plus de 20000 participants, de plusieurs assises et débats et d'une concertation des principales parties prenantes. Cette loi comporte 21 mesures regroupées en quatre thèmes, soit : 1) une priorité : la réussite des étudiants ; 2) une nouvelle ambition pour la recherche ; 3) le décloisonnement comme outil de changement et 4) l'ouverture européenne et internationale.

En lien avec les orientations de cette loi, nous discutons les nouveaux défis pédagogiques qui émergent, en faisant écho aux différences pédagogiques relatives à la formation pharmaceutique en France et au Québec.

\section{Diversification et renouvellement des méthodes pédagogiques}

Le projet de loi français renforce la diversification et le renouvellement des méthodes pédagogiques.

En procédant à la refonte entière du programme de baccalauréat en pharmacie à la Faculté de pharmacie de l'Université de Montréal, le corps professoral et toutes les parties prenantes impliquées ont défini les principales caractéristiques du nouveau programme, à savoir : " un programme [qui] vise d'abord la formation de spécialistes aptes à prodiguer des soins pharmaceutiques de qualité et à jouer pleinement leur rôle dans le système de santé, en étroite collaboration avec les autres intervenants, selon une approche interdisciplinaire ${ }^{[16]}$.

La refonte menée met en évidence le recours à de nombreuses techniques pédagogiques, délaissant 
de façon importante l'enseignement magistral. L'équipe s'est dotée d'un ensemble de techniques (animation, cartographie des connaissances, débat ou panel, démonstration, dialogue socratique écrit, entrevues, étude de cas, exposé interactif, exposé magistral, exposé oral par l'apprenant, jeu de rôle, laboratoire, présentation express, projet, résolution de problèmes complexes, séance d'affichage, séminaire, stage, tournois), qui possèdent les caractéristiques suivantes : 1) l'étudiant est cognitivement actif et très sollicité lors de l'activité ; 2) le travail avec les pairs est possible, sinon essentiel ; 3) la consultation de ressources scientifiques et didactiques de référence est importante ; 4) le contexte professionnel peut servir de toile de fond, de contexte à l'activité proposée ; 5) l'approche pédagogique favorise le développement de l'autonomie ; 6) l'approche pédagogique laisse une place importante à la recherche et à la résolution de problèmes ; 7) le professeur joue le rôle d'accompagnateur, d'expert ou de motivateur.

Les activités d'enseignement et d'apprentissage sélectionnées doivent inciter l'étudiant à une réelle participation et sont choisies principalement en fonction de la nature de la compétence visée, du type d'habiletés à développer et de la nature des connaissances à élaborer.

À titre d'exemple, nous avons comparé les canevas respectifs des programmes de pharmacie de trois universités françaises (Université de Bourgogne Dijon, Université de Limoges, Université d'AixMarseille) et celui de l'Université de Montréal. De façon macroscopique, on note les éléments suivants du renouveau québécois: une intégration des sciences fondamentales aux cours par système physiopathologique, avec insistance sur la pharmacothérapie appliquée, une augmentation du nombre de stages avec exposition clinique proactive auprès des patients, en première, deuxième et quatrième années, représentant le quart de toute la formation, une emphase sur l'apprentissage autodirigé, avec recours à de nombreuses méthodes pédagogiques favorisant des activités asynchrones, une auto-évaluation et une évaluation des connaissances et des compétences de façon précise et concertée.

Une comparaison macroscopique des différents types d'activités d'enseignement et d'apprentissage montre les répartitions suivantes, respectivement en France et au Québec : enseignements magistraux : $60 \%$ vs. $50 \%$; travaux pratiques : $24 \%$ vs. $20 \%$; enseignements dirigés : $15 \%$ vs. $10 \%$; activités d'apprentissage autodirigé : $0 \%$ vs. $30 \%$. Le nombre d'heures de stages est similaire, soit environ 700 heures, pour des programmes respectivement de quatre et six ans (sans tenir compte de formations complémentaires, comme l'internat en France ou la maîtrise en pharmacothérapie avancée au Québec pour exercer en établissement de santé). En outre, les travaux pratiques au Québec ciblent principalement la rédaction de plans de soins pharmaceutiques, la rédaction d'interventions pharmaceutiques, la critique d'articles scientifiques, l'évaluation physique des patients, les discussions de cas, la collaboration interprofessionnelle avec d'autres étudiants en formation (médecins, infirmières, nutritionnistes, etc.) tandis que les travaux pratiques en France ciblent principalement la chimie analytique, la chimie organique, la botanique et la mycologie, la galénique, la physiologie et la pharmacologie, la bactériologie et la virologie, la parasitologie, la biochimie, etc.

\section{L’ère du numérique}

La mesure 8 du projet de loi français prévoit la mise en ligne des enseignements, la promotion des ressources numériques comme outils pédagogiques innovants, l'accompagnement personnalisé des étudiants et la formation à ces nouveaux outils.

Depuis la mise en place du nouveau programme de doctorat professionnel en pharmacie, l'Université de Montréal exige l'acquisition par l'étudiant d'un ordinateur portable uniforme (Macbook Apple) pour l'ensemble des étudiants ; elle offre le déploiement d'un portail pédagogique complet qui comporte $100 \%$ des calendriers pédagogiques, des plans de cours, des contenus pédagogiques, des références documentaires et outils, des évaluations formatives et certificatives. Ce portail assure un accès efficace aux contenus à tous les étudiants et intervenants, afin d'éviter les duplications et d'assurer le maximum de cohérence au fil des enseignements ; il permet aussi l'interactivité en classe (par exemple, sondages en 
lignes, télévoteurs, évaluation proactive des étudiants). En outre, depuis 2010, la faculté utilise une autre plate-forme électronique (Examsoft $\left.{ }^{\circledR}\right)$ pour la rédaction de ses examens en ligne, en classe ${ }^{[17]}$. En France, l'ère du numérique s'amorce mais il n'existe à notre connaissance aucun exemple intégré similaire en pharmacie.

\section{L'étudiant acteur de sa formation}

La mesure 5 du projet de loi prévoit de faire de l'étudiant français l'acteur de sa formation.

Dans le cadre du programme québécois de doctorat professionnel en pharmacie, une majorité des cours sollicitent l'apprentissage autonome et les professeurs/responsables de formation et les tuteurs sont disponibles à date/heure prédéterminée, afin de répondre aux questions et soutenir les étudiants en difficulté. En outre, les activités d'intégration responsabilisent les étudiants dans le cadre de projets qui amènent les étudiants à agir très tôt dans leur carrière comme de véritables professionnels de la santé dans le monde réel. Lors de l'exécution des projets, les étudiants sont confrontés à des difficultés réelles (par exemple, administratives, financières, politiques, pratiques) susceptibles d'être rencontrées dans leur exercice professionnel. En France, il n'existe actuellement pas d'activité d'intégration dans la communauté.

\section{Encadrement des stages et développement en début de cursus}

La mesure 7 de la loi française prévoit l'encadrement des stages et leur développement en début de cursus.

Au Québec, le programme de doctorat professionnel en pharmacie comporte 44 semaines de stages réparties comme suit: un stage de quatre semaines en milieu communautaire dès la première année, un stage de quatre semaines en milieu hospitalier dès la deuxième année, deux stages en milieu communautaire (16 semaines au total) et deux stages en milieu hospitalier (12 semaines au total) et un stage à thématique optionnelle (4 semaines) en quatrième année. En France, bien que le premier stage en milieu communautaire soit possible à la fin de la première année, le stage en milieu hospitalier n'est offert qu'en cinquième année. Si la durée totale des stages est similaire (environ 700 heures en France et au Québec), l'encadrement est différent. Au Québec, l'étudiant est exposé en temps réel, tout le temps, à un pharmacien qui intervient en tant que modèle de rôle dans une relation inter individuelle, tandis qu'en France, compte tenu d'un nombre moins important de pharmaciens au sein des pharmacies hospitalières, l'étudiant interagit moins avec de tels professionnels modèles de rôle.

Les mesures prévues par le gouvernement français sont d'ores et déjà en place au Québec et seraient, à notre sens, bénéfiques à la formation pharmaceutique française.

\section{Conclusion}

Nous pensons que l'adoption du projet de loi pour l'enseignement supérieur et la recherche et l'adoption de la directive européenne relative aux principes communs de formation sont d'excellentes nouvelles pour la pharmacie française. Nous profitons de notre expérience au Québec afin de parfaire notre formation et de contribuer, à notre retour, à cette évolution nécessaire pour répondre aux besoins de nos patients et du système de santé dans lequel nous aurons le privilège d'exercer la pharmacie.

\section{Contributions}

Aurélie Guérin, candidate au Pharm D., a contribué à la formulation de la problématique, à l'extraction des données, à la recherche et à l'analyse documentaires, à la rédaction du manuscrit, à la révision scientifique du contenu et à l'approbation de la version finale.

Marie Élaine Métras, B. Pharm., M.Sc., a contribué à l'extraction des données, à la revue documentaire, à la révision scientifique du contenu et à l'approbation de la version finale. 
Delphine Merger, candidate au Pharm D., a contribué à l'extraction des données, à la revue documentaire, à la révision scientifique du contenu et à l'approbation de la version finale.

Eve Courbon, candidate au Pharm D., a contribué à l'extraction des données, à la revue documentaire, à la révision scientifique du contenu et à l'approbation de la version finale.

Jean-François Bussières, professeur titulaire de clinique en pharmacie, a contribué à la formulation de la problématique, à la recherche et à l'analyse documentaires, à la rédaction du manuscrit, à la révision scientifique du contenu et à l'approbation de la version finale

\section{Déclaration d'intérêts}

Aucun auteur ne déclare de conflit d'intérêt en lien avec le contenu de cet article.

\section{Approbation éthique}

Sans objet

\section{Références}

1. Ministère de l'enseignement supérieur et de la recherche. Projet de loi d'orientation pour l'enseignement supérieur et à la recherche. [On line]. Disponible sur : http://cache.media.enseignementsup-recherche.gouv.fr/file/Loi_ESR_2013/90/7/ dossier-presse-final-projet-loi-esr-mars2013_244907.pdf

2. European parliament and council. Directive 2013/55/ EU of the European parliament and of the council of 20 November 2013 amending Directive 2005/36/EC on the recognition of professional qualifications and regulation (EU) No 1024/2012 on administrative cooperation through the internal market information system. [On line]. Disponible sur : http://eur-lex. europa.eu/LexUriServ/LexUriServ.do?uri=0J: L:2013:354:0132:0170:en:PDF
3. Fielding DW, Brazeau GA, Wasan KM. Introduction to the international pharmacy education supplement. Am J Pharm Educ 2008;72:126.

4. Bourdon O, Ekeland C, Brion F. Pharmacy education in France. Am J Pharm Educ 2008;72:132.

5. Sosabowski MH, Gard PR. Pharmacy education in the United Kingdom. Am J Pharm Educ 2008;72:130.

6. Austin Z, Ensom MH. Education of pharmacists in Canada. Am J Pharm Educ 2008;72:128.

7. Marriott JL, Nation RL, Roller L, Costelloe M, Galbraith K, Stewart P et al. Pharmacy education in the context of Australian practice. Am J Pharm Educ 2008;72:131.

8. Anderson C, Bates I, Beck D, Brock T, Futter B, Mercer $\mathrm{H}$ et al. The WHO UNESCO FIP Pharmacy Education Taskforce: enabling concerted and collective global action. Am J Pharm Educ 2008;72:127.

9. American College of Clinical Pharmacy. Definition of clinical pharmacy. [On line]. Disponible sur : http:/ /www.accp.com/stunet/compass/definition.aspx

10. PharmD Programs - researching your new career options. [On line]. Disponible sur : http:// www.pharmdprograms.org/

11. Elenbaas RM, Worthen DB. Clinical pharmacy in the United States. Transformation of a profession. [On line]. Disponible sur : http://www.accp.com/ bookstore/la_01cpus.aspx

12. Mailhot C, Vinet J, Moreau P, Pharand C, Crevier F, Laurier $\mathrm{C}$ et al. Transformation du programme de baccalauréat en pharmacie de l'Université de Montréal en un programme de doctorat professionnel de 1 er cycle en pharmacie. $1^{\text {ère }}$ partie. Pharmactuel 2006;36:91-7. [On line]. Disponible sur: http://www.pharmactuel.com/sommaires\%5C200602ve.pdf

13. Mailhot C, Vinet J, Moreau P, Pharand C, Crevier F, Laurier $\mathrm{C}$ et al. Transformation du programme de baccalauréat en pharmacie de l'Université de Montréal en un programme de doctorat professionnel de 1er cycle en pharmacie. $2^{\text {ème }}$ partie. Pharmactuel 2006;36:149-55. [On line]. Disponible sur: http://www.pharmactuel.com/sommaires $\% 5$ C200 603ve.pdf 
14. Pharand C, Mallet L, Sheehan NL, Binette MC, Crevier F. Implantation du programme de doctorat professionnel en pharmacie de l'Université de Montréal. Pharmactuel 2009;42(S1):6-10. [On line]. Disponible sur: http://www.pharmactuel.com/ sommaires\%5C2009s1-1.pdf

15. Pharmine. Pharmacy Education in Europe. Country profiles. [On line]. http://www.pharmine.org/ losse_paginas/Country_Profiles/

16. Université de Montréal. Doctorat de premier cycle en pharmacie. [On line]. http://pharm.umontreal.ca/ etudes/premier-cycle/doctorat-de-premier-cycleen-pharmacie-pharm-d/

17. Leclerc G, Moreau P, Bussières JF. Examens assistés par ordinateur l'Udem innove en pharmacie. Québec pharmacie 2013;59:24-26.

Correspondance et offprints : Jean-François Bussières. Département de Pharmacie, Centre hospitalier universitaire Ste-Justine, 3175 Côte Ste-Catherine, Montréal, Québec, H3T 1C5 Canada, Téléphone : 001 (514) 345-4603. Télécopie : 001 (514) 345-4820. Mailto : jf.bussieres@ssss.gouv.qc.ca 